\title{
Click Trains do not Alter Auditory Temporal Order Judgements
}

\author{
Daniel Poole*, Kyle J. Lees and Luke A. Jones \\ Faculty of Biology Medicine and Health Manchester, The University of Manchester, \\ Manchester, UK
}

Received 26 November 2019; accepted 7 May 2020

\begin{abstract}
Brief periods of repetitive stimulation (click trains) presented either contiguous or simultaneous to an interval have been previously shown to impact on its perceived duration. In the current investigation we asked whether the perception of temporal order can be altered in a similar way. Participants completed a dichotic spectral temporal order judgement task with the stimuli titrated to their individual thresholds. Immediately prior to the judgement, participants were presented with five seconds of click trains, white noise or silence. We extended previous work on this topic by using each participant's accuracy and response time data to estimate diffusion model parameters so that the cognitive mechanisms underlying any effect of click trains on the response could be disentangled. There was no effect of stimulation condition on participant's accuracy, or diffusion model parameters (drift rate, boundary separation or non-decision time). The present findings therefore suggest that click trains do not influence temporal order perception. Additionally, the previous suggestion that click trains induce an increase in the rate of information processing was not supported for this temporal order task. Further work probing the limits and conditions of the click train effect will help to constrain and extend theoretical accounts of subjective timing.
\end{abstract}

\section{Keywords}

Temporal order judgement, timing, click trains, drift diffusion model

\section{Introduction}

An interesting finding from experimental studies of human timing is that subjective time can be influenced by factors unrelated to the timing judgement. Treisman et al. (1990) discovered that judgements of duration could be altered by

\footnotetext{
* To whom correspondence should be addressed. E-mail: daniel.poole@manchester.ac.uk 
the presentation of repetitive stimulation. Treisman et al. used trains of auditory clicks presented at the same time as the to-be-timed stimulus. The presentation of this stimulation has the effect of making estimations of stimulus duration relatively longer, and production of intervals relatively shorter. However, the important trademark of this effect is that it is multiplicative. The degree of overestimation (or underproduction) increases as the duration to be estimated/produced increases. In other words, if one plots real duration against estimated duration for the click condition and for the control (no-click condition) then there will be a significant difference in the slope of the two functions. These slope effects are consistent with the idea that humans possess an internal clock of a pacemaker/ accumulator type, and that these repetitive stimulations speed up this pacemaker.

Later studies found that the click trains could be presented before the stimulus to be timed (Burle \& Casini, 2001; Droit-Volet, 2010, 2017; Penton-Voak et al., 1996; Wearden et al., 1998, 1999, 2009, 2017), and that repetitive visual stimulation (a series of flashes) also produces the same effect (Droit-Volet, 2010; Droit-Volet \& Wearden, 2002; Ortega \& López, 2008; Treisman \& Brogan, 1992; Wearden et al., 2017). These studies have found the click train or flicker effect across many different timing tasks that require a judgement or production of a stimulus duration including: verbal estimation (requiring the participant to quantify the duration of a stimulus), temporal generalization (comparing a duration with a previously remembered duration), discrimination (deciding which of two tones was longer), temporal reproduction (reproducing a duration) and temporal bisection (deciding if a duration is more similar to a remembered long or short duration). This is consistent with the idea that performance on all of these tasks is underpinned by the internal clock/pacemaker which is affected by the click trains. Click trains (and flickers) have also been found to have an effect on non-temporal tasks requiring either a fast reaction time response, or the encoding of information from briefly presented stimuli (Jones et al., 2011, Wearden et al., 2017), and on the perception of quantity and line length (if presented sequentially, see DroitVolet, 2010). This implies that the effect may have some general impact on the rate of information processing, which could theoretically be related to the pacemaker.

Although the click train effect has been observed across a variety of tasks, divergent findings have been reported. Tactile vibration trains lead to overestimation of intervals, but the effect is additive (i.e., a constant proportion) rather than multiplicative (Jones \& Ogden, 2017). An additive effect has also been observed when making duration judgements about moving visual stimuli following both click trains and white noise (Makin et al., 2012). When the stimulation is presented immediately following the interval, click trains/flicker of increasing frequency leads to a subjective reduction in subjective duration (Ono \& Kitazawa, 2010, 2011). A study of timing of unfilled intervals did not show any effects of click trains presented prior to, during or after the interval (Repp et al., 2013). Finally, verbal estimations of the duration of symmetrical and random patterns were found to be 
comparable between click trains and white noise (although durations in the click train condition were judged longer than when preceded by silence; Palumbo et al., 2015). As the click train effect thus appears to be highly dependent on the nature of the timing judgement and the presentation of the click trains, a fruitful line of inquiry is for experimental studies probing the conditions under which click trains impact on time perception. In the present study, we considered the impact of click trains on relative timing, which is currently unknown, using the temporal order judgement task.

In the temporal order judgement task two stimuli are presented in quick succession and the participant is asked to judge their order. Performance is measured for stimuli presented at a range of stimulus onset asynchronies (SOA, the difference in time between the onset of the respective stimuli). At greater SOA participants are better able to discriminate the relative order, whereas at shorter SOA the stimuli become more difficult to parse. There is a substantial body of work using both crossmodal and unimodal Temporal Order Judgements (TOJs) to measure temporal acuity for a variety of stimuli (e.g., Hirsh \& Sherrick, 1961; Jaskowski et al., 1990; Kanabus et al., 2002; Smith, 1933; Spence et al., 2001). Auditory TOJs are of particular relevance to the current investigation. Typically, auditory TOJs involve the participant responding to tones which differ in spatial location (presented dichotically) and/or spectrally (different frequencies), with thresholds typically lower using the spectral paradigm (Fostick \& Babkoff, 2017; Fostick et al., 2011; Szymaszek et al., 2009). Thresholds can be influenced by properties of the stimuli. For instance, increasing tone duration increases participant thresholds (Babkoff \& Fostick, 2013). Auditory TOJ thresholds are increased in a number of groups including elderly (Szelag et al., 2018), dyslexic (Fostick, 2012) and autistic (Kwakye et al., 2011) people. Previous work has revealed that TOJ acuity can be affected by non-task-relevant stimuli. A performance benefit is observed to TOJ stimuli presented near threshold when crossmodal stimuli are presented in near co-incidence (Hairston et al., 2005, 2006; Keetles \& Vroomen, 2008; MoreinZamir et al., 2003).

It has been theorised that perception occurs within a functional moment in the range of tens of milliseconds (Pöppel, 1997, 2009; Pöppel \& Bao, 2014; Wittmann, 2011). The duration of the functional moment determines how perceptual order is perceived. Temporally (and spatially) separated sensory information occurring within this duration are integrated into a single perceptual event making it impossible to discriminate the relative order. Neural oscillations triggered by stimulus activity are believed to control this process with each cycle defining the duration of the functional moment. The rate of these oscillations could be controlled by the pacemaker of the internal clock with higher pacemaker rates leading to shorter temporal oscillations (Szelag et al., 2004). In a previous study, factor analysis revealed that TOJ thresholds loaded onto a common factor with measures of performance on interval judgement tasks (discrimination thresholds and responses 
on a temporal generalisation experiment) suggesting that these judgements are controlled by a common mechanism (Rammsayer \& Brandler, 2004). Any manipulation increasing the rate of the pacemaker (such as click trains) should improve acuity to relative order, the reduced duration of the functional moment leading to less integration of stimuli.

To explore any effect of click trains on TOJs, participants in the present study completed spectral judgements to stimuli presented dichotically. Participants completed TOJs immediately following one of three stimulation conditions; $5 \mathrm{~Hz}$ click train, silence or white noise. TOJ stimuli were titrated to the participant's threshold to constrain performance between individuals before introducing the stimulation condition. If click trains impact on temporal integration as described above, then accuracy will be increased in comparison with white noise and silence. On the other hand, it may be that click trains lead to a general increase in the sampling rate of stimulus information (Jones et al., 2011). To explore this possibility, we aimed to further unpack the mechanisms underlying any effect of click trains on perception by estimating diffusion model parameters in each condition.

The diffusion model framework describes error and response time (RT) on a two-choice task as the result of combination of cognitive processes (Heitz, 2014; Ratcliff \& McKoon, 2008; Voss et al., 2013). Each response is represented by a boundary and following the presentation of the stimulus, a noisy process of evidence accumulation occurs with a response triggered once sufficient evidence is accumulated in order to reach a given boundary. Fitting the diffusion model to RT and accuracy data allows a number of parameters to be estimated which provide greater power to observe effects and better constrain theories of the interpretation of the cognitive processes which underlie the response (Ratcliff et al., 2016a). Relevant to the current investigation are drift rate $(v)$, boundary separation $(\alpha)$ and non-decision time $\left(t_{0}\right)$. Drift rate is the average of the evidence accumulation process and is determined by the quality of the information extracted from the stimulus. A higher drift rate implies the quality of the information extracted from the information is increased (Voss et al., 2015) and the diffusion process will reach the correct boundary more rapidly leading to faster and more accurate responses. The boundary separation is a measure of speed-accuracy trade-off effects (Ratcliff et al., 2016b). Increased values represent more conservative response criteria as more time is required until the diffusion process will reach a boundary, the likelihood of the incorrect boundary being triggered is reduced and the response will be slower, but more accurate. The processes outside of the decision-making process which comprise the participant's response are captured in the non-decision time parameter. This is believed to include the time taken for the stimulus to be encoded and preparation of the movement to execute the response. It is valuable to decompose RT and accuracy into these different processes as any RT or accuracy difference might be the consequence of any of these processes. If a general increase in the rate of information processing is induced by the click trains, then 
there will be an increase in drift rates and reduction in non-decision time relative to the white noise and silence conditions.

\section{Methods}

\subsection{Participants}

Sixty participants ( 16 male) with a mean age of 21.33 years \pm 3.42 were recruited via advertisements at the University of Manchester. Participants were compensated with course credit, or a small amount of cash. All participants gave written consent and the study was approved by the University of Manchester's ethics committee in accordance with the Declaration of Helsinki.

\subsection{Apparatus}

Auditory stimuli were delivered through Sennheiser HD 65 TV headphones controlled by E-Prime 1.2 software (Psychology Software Tools Inc., Pittsburgh, PA, USA). For the TOJ task, low tones $(440 \mathrm{~Hz}, 0.8 \mathrm{~A})$ were presented to the left ear and high tones $(600 \mathrm{~Hz}, 0$. A were presented to the right ear. The duration of the first tone was always $500 \mathrm{~ms}$ and the second tone varied in duration at each SOA. This means that the SOA varied, but stimulus offset was always identical. In the experimental task there were three stimulation conditions: click trains, white noise and silence. Click train stimuli comprised 1.0-ms clicks presented at a rate of 5 $\mathrm{Hz}$ for $5 \mathrm{~s}$. White noise $(0.8 \mathrm{~A})$ was presented for $5 \mathrm{~s}$. Each stimulation condition was preceded by a chirp ramped from $440 \mathrm{~Hz}$ to $1320 \mathrm{~Hz}$, which was reversed for a second chirp presented at the end of the period of stimulation. Responses were recorded using the number pad on the computer keyboard with the participant instructed to press ' 8 ' if the high tone was presented first and ' 2 ' for the low tone.

\subsection{Procedure}

\subsubsection{Threshold}

Prior to the experimental task the participants completed an adaptive staircase procedure (cf. Hairston \& Maldjian, 2009) to determine their approximate threshold on the TOJ task. Firstly, the participant was familiarised with the stimuli. The high and low tone were presented for $500 \mathrm{~ms}$ alongside a visual prompt indicating whether the tone was low or high. To confirm they could distinguish the tones, the participant then completed six trials in which they were asked to identify whether the high or low tone was presented. The participants then completed ten TOJ trials with the SOA at $150 \mathrm{~ms}$ to confirm they understood the task instructions. They were required to perform at $80 \%$ accuracy to continue to the next stage. The participants then began the staircase procedure. The SOA of the tones was adjusted according to a 3-down/1-up rule (Kaernbach, 1991) converging on 75\% accuracy. There were two interleaved staircases. Each staircase began at an SOA of $100 \mathrm{~ms}$ and the initial step size was $10 \mathrm{~ms}$, which was reduced to $5 \mathrm{~ms}$ after five steps 
and $1 \mathrm{~ms}$ after nine steps. The procedure terminated after 16 reversals and the threshold was calculated as the average SOA of the final six reversals. The staircase producing the lowest threshold estimate was used to set the stimuli in the experimental task.

\subsubsection{Experimental procedure}

After completing the threshold procedure, the participants continued to the experimental task. TOJ stimuli were presented at the participant's threshold, or at the SOA $\pm 25 \%$ threshold. Stimuli of each SOA were randomised in each block. It was anticipated that including stimuli above and below the threshold would prevent the participant becoming familiarised with the TOJ stimuli and changing response strategy during the experimental task. Each stimulation condition (click trains, white noise or silence) was presented in separate blocks with each block in a random order before repeating a second block of each condition in a random order. After the second chirp marking the end of the stimulation period there was a pause of $500-1000 \mathrm{~ms}$ drawn from a uniform distribution. A trial schematic is displayed in Fig. 1.

Each block contained 42 trials ( 14 trials at each SOA) giving 84 trials in each stimulation condition and 252 trials in total. Participants were encouraged to take a short break between each block. In the experimental task participants were instructed to respond to the order of the stimuli 'as quickly and as accurately as possible'. There were no specific instructions on whether the participant should use one or two hands to respond. Prior to beginning the experimental trials, participants completed a practice block of 30 trials in the silence condition with 10 trials at each SOA to familiarise themselves with the experimental procedure. The entire testing session lasted approximately $1 \mathrm{~h} 30 \mathrm{~min}$.

\subsubsection{Analysis}

Accuracy. Accuracy data in each condition were calculated. Trials with RT $<100$ $\mathrm{ms}$ ( $14.80 \%$ of all trials), or $>2000 \mathrm{~ms}$ ( $3.50 \%$ of trials) were removed from analysis. It was assumed that these reflected anticipation errors and misses. Participant's accuracy was averaged across each SOA and a within-participants ANOVA was then used to compare accuracy in each stimulation condition [Click Trains, White Noise, Silence]. Alongside frequentist analysis, we also calculated the Bayes Factor for comparisons between click train and the silence and white noise conditions using a within-participants Bayesian $t$-test using JASP (JASP team, 2019) with the default prior setting. The Bayes Factor $\left(\mathrm{BF}_{01}\right)$ describes the extent to which the data support the null hypothesis (no differences between the conditions) relative to the alternative (data from each condition drawn from different distributions).

Drift diffusion modelling. Prior to analysis, within-participant outliers of RT in each condition were removed using the non-recursive procedure described by Van Selst and Jolicoeur (1994) using the trimr package (Grange, 2015). To prevent the impact of very fast RT on the calculation of model parameters trials with RTs $<200$ ms were removed. Diffusion model parameters were estimated separately 


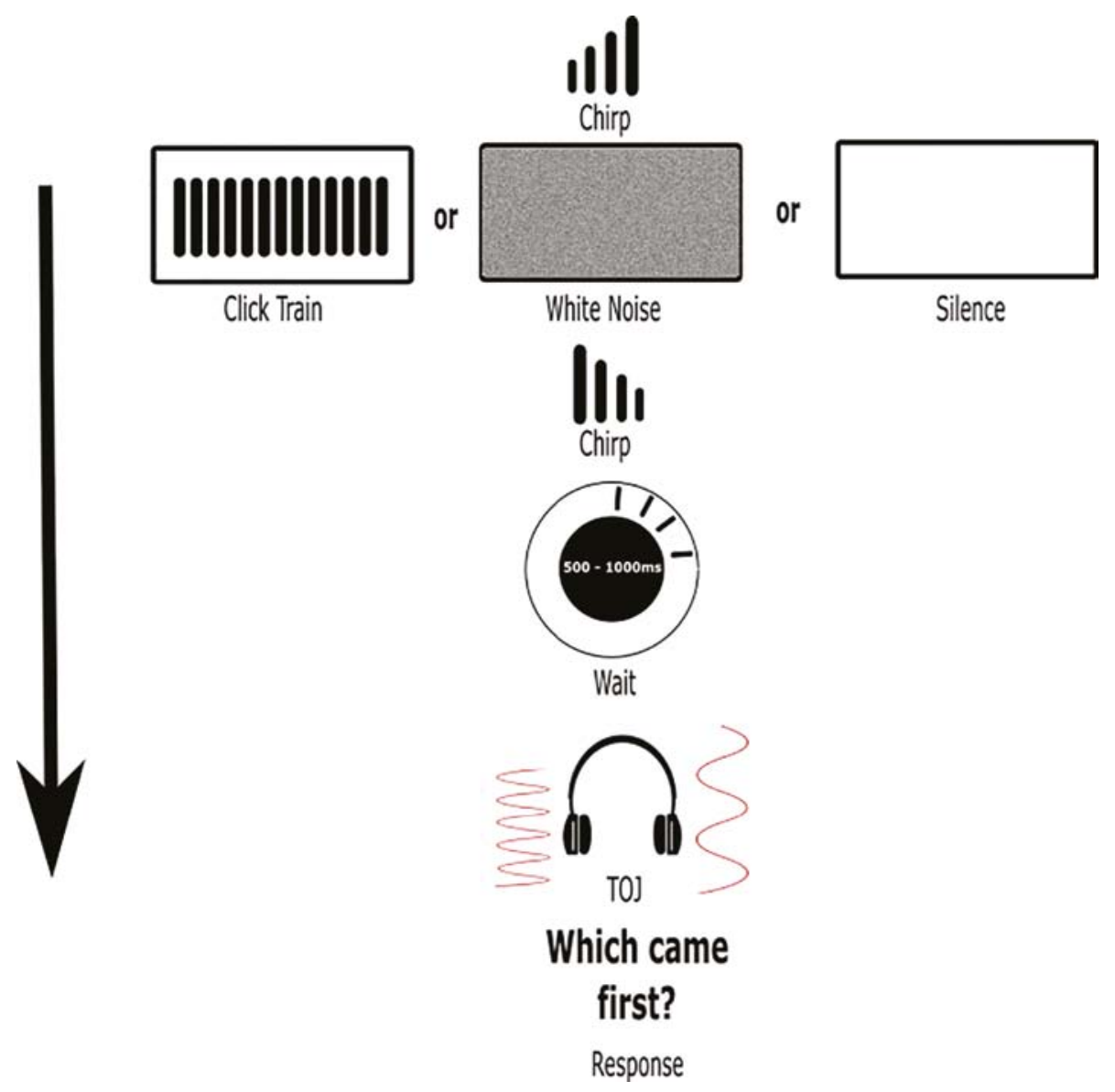

Figure 1. Schematic of a trial in the experimental task.

for each individual for each stimulation condition using the maximum likelihood procedure as implemented in fast- $d m$-30 (Voss et al., 2015). Three parameters were left free to vary in the fitting procedure: drift rate $(v)$, boundary separation $(\alpha)$ and non-decision time $\left(t_{0}\right)$. As the boundaries corresponded to correct and error response, and it is not possible to have a bias towards responding correctly or erroneously in advance of a trial, the starting point was fixed at 0.5 . The intertrial variability of start point and drift rate were set to zero in line with previous recommendations (Lerche \& Voss, 2016). To assess model fits of the data, the fast$d m$-30 construct-samples function was used to simulate 1000 predicted RTs for each participant's estimated model parameters in each condition. The cumulative distribution functions of the predicted RTs overlaid those measured for each participant, suggesting models provided an excellent fit of the data (see the supplementary resources file on Open Science Framework). 


\section{Results}

\subsection{Threshold}

Participant's average threshold was $103.73 \mathrm{~ms}( \pm 47.62)$ SOA.

\subsection{Accuracy}

Accuracy data in each condition are displayed in Fig. 2.

There were no statistical differences between accuracy in the different stimulation conditions. To correct negative skew $(-1.21)$, the data were reflected and base10 log-transformed for analysis. An ANOVA with a within-participants factor of Condition [Click Trains, Silence, White Noise] revealed no main effect of Condition $\left(F_{2,118}=0.63, p=0.534, \eta_{\mathrm{p}}{ }^{2}=0.011\right)$. The Bayes Factor and an estimate of effect size for comparisons of click trains with silence and white noise are given in Table 1.

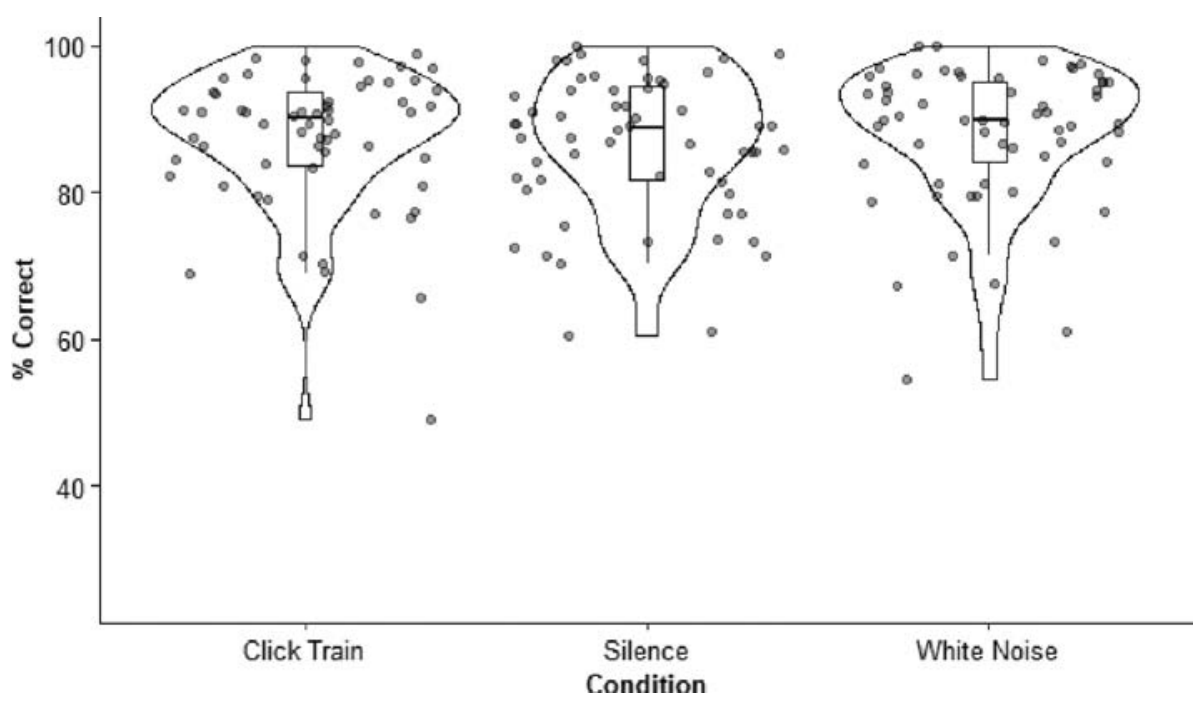

Figure 2. Combined box-violin plots displaying average accuracy across the three stimulus onset asynchronies in each condition. The datapoints give the individual participant's average for that condition. Note the analysis was conducted on transformed data.

Table 1.

Effect size (Cohen's $d$ including 95\% confidence intervals) and Bayes Factors for comparisons of accuracy following silence and white noise in comparison with click trains. Effect sizes were small and the Bayes Factor suggested strong evidence in favour of the null.

$\begin{array}{lll}\text { Clicks Trains vs } & d[95 \% \mathrm{CI}] & \mathrm{BF} 01\end{array}$

Silence $\quad 0.04[-0.22,0.29] \quad 6.79$

White noise $\quad-0.11[-0.37,0.14] \quad 4.93$ 


\subsection{Diffusion model parameters}

Response time distributions are displayed in Fig. 3. Estimated drift rate $(v)$, boundary separation $(\alpha)$ and non-decision time $\left(t_{0}\right)$ parameters are displayed in Fig. 4.

There were no statistical differences between any of the parameter estimates for any stimulation conditions. Within-participants ANOVAs revealed no main effect of Condition for drift rates $\left(F_{2,118}=1.20, p=0.140, \eta^{2}=0.033\right)$, boundary separation $\left(F_{2,118}=0.26, p=0.770, \eta^{2}=0.004\right)$, or non-decision time $\left(F_{2,118}=\right.$ 2.13, $\left.p=0.123, \eta^{2}=0.035\right)$. The Bayes Factor and an estimate of effect size for comparisons of click trains with silence and white noise for each parameter estimate are given in Table 2.
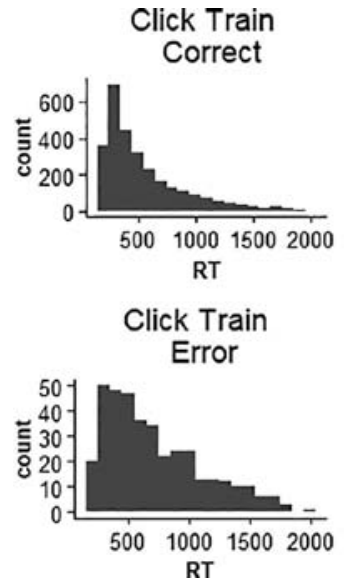

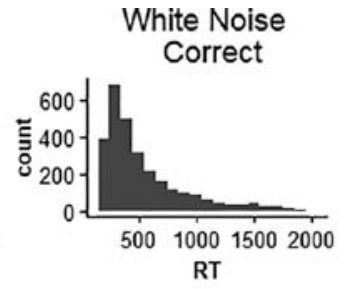

White Noise

Error

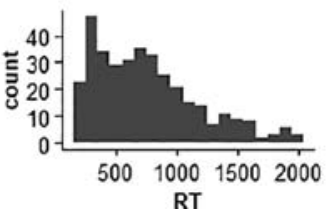

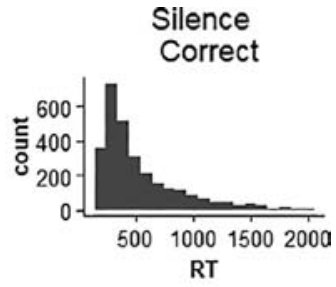

Silence

Error

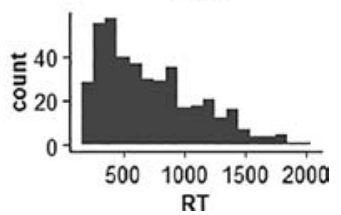

Figure 3. Distribution of response times (RT) across all participants in each condition for correct and error trials.
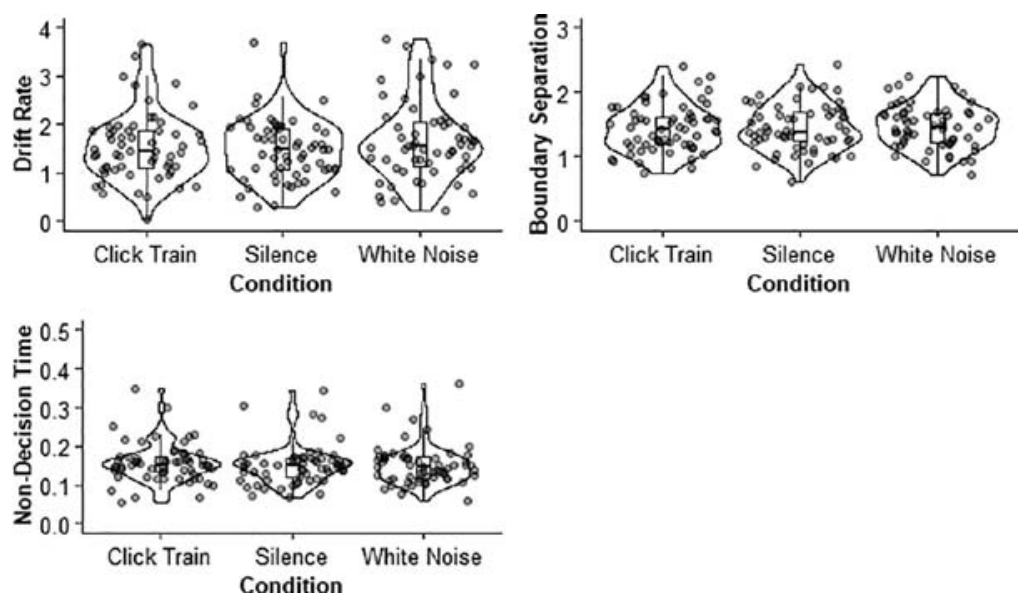

Figure 4. Combined box-violin plots displaying estimated diffusion model parameters in each condition. Points give the individual participant's parameter estimate for that condition. 
Table 2.

Effect size (Cohens d including 95\% confidence intervals) and Bayes Factors for comparisons of drift rate, boundary separation and non-decision time following silence and white noise in comparison with click trains. Effect sizes were small and Bayes Factor suggested strong evidence in favour of the null for drift rates and boundary separation and anecdotal evidence in favour of the null for non-decision times.

\begin{tabular}{|c|c|c|c|c|c|c|}
\hline \multirow[t]{2}{*}{ Clicks trains vs } & \multicolumn{2}{|l|}{ Drift rate } & \multicolumn{2}{|c|}{ Boundary separation } & \multicolumn{2}{|c|}{ Non-decision time } \\
\hline & $d[95 \% \mathrm{CI}]$ & $\mathrm{BF}_{\mathrm{ol}}$ & $d[95 \% \mathrm{CI}]$ & $\mathrm{BF}_{\mathrm{ol}}$ & $d[95 \% \mathrm{CI}]$ & $\mathrm{BF}_{\mathrm{ol}}$ \\
\hline Silence & $\begin{array}{l}0.12 \\
{[-0.13,0.38]}\end{array}$ & 4.65 & $\begin{array}{l}-0.10 \\
{[-0.36,0.16]}\end{array}$ & 6.87 & $\begin{array}{l}0.18 \\
{\left[\begin{array}{ll}-0.08 & 0.44\end{array}\right]}\end{array}$ & 2.84 \\
\hline White noise & $\begin{array}{l}-0.13 \\
{[-0.39,0.12]}\end{array}$ & 4.28 & $\begin{array}{l}-0.03 \\
{\left[\begin{array}{ll}-0.29 & 0.22\end{array}\right]}\end{array}$ & 5.35 & $\begin{array}{l}0.24 \\
{\left[\begin{array}{ll}-0.02 & 0.5\end{array}\right]}\end{array}$ & 1.36 \\
\hline
\end{tabular}

\section{Discussion}

In the present study, participants completed spectral TOJs to dichotic stimuli which were preceded by click trains, white noise or silence. There were no statistically significant differences in accuracy between the stimulation conditions, nor in estimated diffusion model parameters: drift rate, boundary separation and nondecision time were all comparable between the conditions. As such, the current investigation suggests that click trains do not impact upon the perception of auditory TOJs, nor on the decision processes associated with these judgements. We consider possible explanations of this null effect which highlight ways in which this finding extends current understanding of the click train effect.

The first, and most parsimonious, explanation of this null effect is that click trains simply do not impact temporal order perception. The null finding in the current study has added to the body of work on the click train effect, providing valuable insight into the nature of timing tasks which are influenced by click trains. Previous work indicated that click trains do not impact on duration judgements of unfilled intervals (Repp et al., 2013). Click trains may only alter the perception of the duration of continuous stimuli (as used by Droit-Volet \& Wearden, 2002; Penton-Voak et al., 1996 amongst others). Contrary to our hypothesis, the current findings suggest that click trains do not impact on temporal integration and this could be interpreted as evidence against the suggestion that TOJs are controlled by an internal clock mechanisms common with interval timing (Rammsayer \& Brandler, 2004).

In previous work, click train effects have been observed for non-timing tasks (Droit-Volet, 2010; Jones et al., 2011). Click trains were found to induce faster RT 
on a simple response task to multiple items, a speeded mental arithmetic task, recall of letters in a memory task, recognition on a masking task and magnitude estimates of line length and quantity (see Note 1). It has been proposed that click trains increase the rate of information processing, meaning that the quantity of information which can be processed is increased. The data in the present study do not support the previous suggestion that the rate of information processing is increased following the presentation of click trains, at least for TOJs. Drift rates are an estimate of the quality of information extracted from a stimulus and nondecision time includes all non-decision-related processes, including the encoding of the stimulus (Voss et al., 2015; White et al., 2016). If the presentation of click trains increased information processing then it is likely an increase in estimates of participants' drift rates and reduction in non-decision time would be observed. The click stimuli used in the present study were similar to those used by Jones et al. (2011) and as such the question of why the previous study observed effects consistent with increased rate of information processing on these non-temporal tasks, but no effect was observed for timing judgements in the present study, is intriguing.

A second, more speculative explanation is that click trains may have had some impact on the perception of the stimuli, but the design of the current study did not allow for this effect to be observed. For instance, it could be that the click trains led to a subjective lengthening of both successive auditory stimuli (presumably if only the first stimulus presented was lengthened, this would have made them more difficult to parse and thus led to a detriment in performance). The clicks were presented binaurally before the participant made judgements about the relative order of dichotic tones. As the tones were presented at the participant's threshold the SOA was very short and the click train effect may have persisted beyond the onset of the first stimulus leading to a subjective lengthening of both the tones (although see, Babkoff \& Fostick, 2013, who showed increasing tone durations reduced TOJ performance). If we assume that the click trains only impact on the first stimulus to arrive following the clicks, then this account could plausibly be tested in future work using a monoaural spectral TOJ task with clicks and TOJ stimuli presented to a single channel. The nature of click train effects are not well understood, and a programme of work systematically manipulating the properties and presentation of the stimuli is required to constrain the theory underlying its putative effects.

In the present study we used a manipulation which has previously been shown to impact on interval judgements across a range of timing tasks on an auditory TOJ task. There was no effect on the accuracy of TOJ judgements, nor on drift diffusion parameters providing a measure of information processing and decision making. These findings suggest that click trains do not have a generalised effect on timing or information processing. Future work is required to determine the precise conditions under which click trains influence the perception of stimuli. 


\section{Supplementary Material}

\section{Data and materials can be found on the Open Science Framework: https://osf.io/y4kp9/}

\section{Note}

1. The effect on line length and quantity only occurs if presented sequentially (see Droit-Volet, 2010).

\section{References}

Babkoff, H., \& Fostick, L. (2013). The role of tone duration in dichotic temporal order judgment. Atten. Percept. Psychophys., 75, 654-660. doi: 10.3758/s13414-013-0449-6.

Burle, B., \& Casini, L. (2001). Dissociation between activation and attention effects in time estimation: Implications for internal clock models. J. Exp. Psychol. Hum. Percept. Perform., 27, 195-205. doi: 10.1037//0096-1523.27.1.195.

Droit-Volet, S. (2010). Speeding up a master clock common to time, number and length? Behav. Proc., 85, 126-134. https://doi.org/10.1016/j.beproc.2010.06.017.

Droit-Volet, S. (2017). Time dilation in children and adults: The idea of a slower internal clock in young children tested with different click frequencies. Behav. Proc., 138: 152-159. https://doi .org/10.1016/j.beproc.2017.03.005.

Droit-Volet, S., \& Wearden, J. (2002). Speeding up an internal clock in children? Effects of visual flicker on subjective duration. Q. J. Exp. Psychol., 55(3), 37-41. https://doi.org/10.1080/ 02724990143000252.

Fostick, L. (2012). Auditory temporal processing as a specific deficit among dyslexic readers. Psychol. Res., 2(2), 77-88.

Fostick, L., \& Babkoff, H. (2017). Auditory spectral versus spatial temporal order judgment: Threshold distribution analysis. J. Exp. Psychol. Hum. Percept. Perform., 43, 1002-1012. https://doi .org/10.1037/xhp0000359.

Fostick, L., Ben-Artzi, E., \& Babkoff, H. (2011). Stimulus-onset-asynchrony as the main cue in temporal order judgment. Audiol. Res., 1, e5. https://doi.org/10.4081/audiores.2011.e5.

Fostick, L., Bar-El, S., \& Ram-Tsur, R. (2012). Auditory temporal processing as a specific deficit among dyslexic readers. Psychol. Res., 2(2), 77-88.

Grange, J. (2015). trimr: An implementation of common response time trimming methods. R package. Available at: http://CRAN.R-project.org/package=trimr.

Hairston, W. D., \& Maldjian, J. A. (2009). An adaptive staircase procedure for the E-Prime programming environment. Comput-Methods Programs Biomed., 93, 104-108. https://doi.org/10.1016/j .cmpb.2008.08.003.

Hairston, W. D., Burdette, J. H., Flowers, D. L., Wood, F. B., \& Wallace, M. T. (2005). Altered temporal profile of visual-auditory multisensory interactions in dyslexia. Exp. Brain Res., 166, 474-480. https://doi.org/10.1007/s00221-005-2387-6.

Hairston, W. D., Hodges, D. A, Burdette, J. H., \& Wallace, M. T. (2006). Auditory enhancement of visual temporal order judgment. Neuroreport, 17, 791-795. https://doi.org/10.1097/01 .wnr.0000220141.29413.b4. 
Heitz, R. P. (2014). The speed-accuracy tradeoff: history, physiology, methodology, and behavior. Front. Neurosci., 8, 150. https://doi.org/10.3389/fnins.2014.00150.

Hirsh, I. J., \& Sherrick Jr, C. E. (1961). Perceived order in different sense modalities. J. Exp. Psychol., 62, 423-432. doi: 10.1037/h0045283.

Jaśkowski, P., Jaroszyk, F., \& Hojan-Jezierska, D. (1990). Temporal-order judgments and reaction time for stimuli of different modalities. Psychol. Res., 52, 35-38. doi: 10.1007/BF00867209.

JASP Team (2019). JASP version 0.11.1. Available at https://jasp-stats.org.

Jones, L. A., \& Ogden, R. S. (2017). Vibrotactile timing: Are vibrotactile judgements of duration affected by repetitive stimulation? Q. J. Exp. Psychol., 69, 75-88. https://doi.org/10.1080/1747021 8.2015.1023735.

Jones, L. A., Allely, C. S., \& Wearden, J. H. (2011). Click trains and the rate of information processing: Does "speeding up" subjective time make other psychological processes run faster? Q.J. Exp. Psychol., 64, 363-380. https://doi.org/10.1080/17470218.2010.502580.

Kaernbach, C. (1991). Simple adaptive testing with the weighted up-down method. Percept. Psychophys., 49, 227-229.

Kanabus, M., Szeląg, E., Rojek, E., \& Pöppel, E. (2002). Temporal order judgement for auditory and visual stimuli. Acta Neurobiol. Exp., 62, 263-270.

Keetles, M., \& Vroomen, J. (2008). Tactile-visual temporal ventriloquism: No effect of spatial disparity. Percept. Psychophys., 70, 765-771. https://doi.org/10.3758/PP.70.5.765.

Kwakye, L. D., Foss-Feig, J. H., Cascio, C. J., Stone, W. L., \& Wallace, M. T. (2011). Altered auditory and multisensory temporal processing in autism spectrum disorders. Front. Integr. Neurosci., 4, 129. https://doi.org/10.3389/fnint.2010.00129.

Lerche, V., \& Voss, A. (2016). Model complexity in diffusion modeling: benefits of making the model more parsimonious. Front. Psychol., 7, 1324. https://doi.org/10.3389/fpsyg.2016.01324.

Makin, A. D. J., Poliakoff, E., Dillon, J., Perrin, A., Mullet, T., \& Jones, L. A. (2012). The interaction between duration, velocity and repetitive auditory stimulation. Acta Psychol., 139, 524-531. https://doi.org/10.1016/j.actpsy.2012.01.013.

Morein-Zamir, S., Soto-Faraco, S., \& Kingstone, A. (2003). Auditory capture of vision: examining temporal ventriloquism. Cogn. Brain Res., 17, 154-163. https://doi.org/10.1016/S0926 $-6410(03) 00089-2$.

Ono, F, \& Kitazawa, S. (2010). Shortening of subjective tone intervals followed by repetitive tone stimuli. Atten. Percept. Psychophys., 72, 492-500. https://doi.org/10.3758/APP.72.2.492.

Ono, F., \& Kitazawa, S. (2011). Shortening of subjective visual intervals followed by repetitive stimulation. PloS ONE, 6, e28722. https://doi.org/10.1371/journal.pone.0028722.

Ortega, L. \& López, F. (2008). Effects of visual flicker on subjective time in a temporal bisection task. Behav. Proc., 78, 380-386. https://doi.org/10.1016/j.beproc.2008.02.004.

Palumbo, L., Ogden, R., Makin, A. D. J., \& Bertamini, M. (2015). Does Preference for Abstract Patterns Relate to Information Processing and Perceived Duration? I-Perception, 6, 20416695156043, https://doi.org/10.1177/2041669515604436.

Penton-Voak, I. S., Edwards, H., Percival, A., \& Wearden, J. H. (1996). Speeding up an internal clock in humans? Effects of click trains on subjective duration.J. Exp. Psychol. Anim. Behav. Proc., 22, 307-320. https://doi.org/10.1037/0097-7403.22.3.307.

Pöppel, E. (1997). A hierarchical model of temporal perception. Trends Cogn. Sci., 1, 56-61. https:// doi.org/10.1016/S1364-6613(97)01008-5.

Pöppel, E. (2009). Pre-semantically defined temporal windows for cognitive processing. Philos. Trans. R. Soc. B Biol. Sci., 364(1525), 1887-1896. https://doi.org/10.1098/rstb.2009.0015. 
Pöppel, E., \& Bao, Y. (2014). Temporal windows as a bridge from objective to subjective time. In V. Arstila, \& D. Lloyd (Eds), Subjective Time: The Philosophy, Psychology, and Neuroscience of Temporality, (pp. 241-262). MIT Press Direct, Cam,bridge, MA, USA. https://doi.org/10.7551/ mitpress/8516.003.0020.

Rammsayer, T. H., \& Brandler, S. (2004). Aspects of temporal information processing: A dimensional analysis. Psychol. Res., 69, 115-123. https://doi.org/10.1007/s00426-003-0164-3.

Ratcliff, R., \& McKoon, G. (2008). The diffusion decision model: theory and data for two-choice decision tasks. Neural Comput., 20, 873-922. https://doi.org/10.1162/neco.2008.12-06-420.

Ratcliff, R., Thompson, C. A., \& McKoon, G. (2016a). Modelling individual differences in response time and accuracy in numeracy. Cognition, 137, 115-136. https://doi.org/10.1016/j.cognition .2014.12.004.Modeling.

Ratcliff, R., Smith, P. L., Brown, S. D., \& McKoon, G. (2016b). Diffusion decision model: current issues and history. Trends Cogn. Sci., 20, 260-281. https://doi.org/10.1016/j.tics.2016.01.007.

Repp, B. H., Mendlowitz, H. B., \& Hove, M. J. (2013). Does rapid auditory stimulation accelerate an internal pacemaker? Don't bet on it. Timing Time Percept., 1, 65-76. https://doi.org/10.1163/ 22134468-00002001.

Smith, W. F. (1933). The relative quickness of visual and auditory perception. J. Exp. Psychol., 16, 239-257. https://doi.org/10.1037/h0071379.

Spence, C., Shore, D. I., \& Klein, R. M. (2001). Multisensory prior entry. J. Exp. Psychol. Gen., 130, 799-832. https://doi.org/10.1037/0096-3445.130.4.799.

Szelag, E., Kanabus, M., Kolodziejczyk, I., Kowalska, J., \& Szuchnik, J. (2004). Individual differences in temporal information processing in humans. Acta Neurobiol. Exp., 64, 349-366.

Szelag, E., Jablonska, K., Piotrowska, M., Szymaszek, A., \& Bednarek, H. (2018). Spatial and spectral auditory temporal-order judgment (TOJ) tasks in elderly people are performed using different perceptual strategies, Front. Psychol., 9, 2557. https://doi.org/10.3389/fpsyg.2018.02557.

Szymaszek, A., Sereda, M., Pöppel, E., \& Szelag, E. (2009). Individual differences in the perception of temporal order: The effect of age and cognition. Cogn. Neuropsychol., 26, 135-147. https://doi .org/10.1080/02643290802504742.

Treisman, M., \& Brogan, D. (1992). Time perception and the internal clock: Effects of visual flicker on the temporal oscillator. Eur. J. Cogn. Psychol., 4, 41-70. https://doi.org/10.1080/ 09541449208406242.

Treisman, M., Faulkner, A., Naish, P. L. N., \& Brogan, D. (1990). The internal clock: evidence for a temporal oscillator underlying time perception with some estimates of its characteristic frequency. Perception, 19, 705-742. https://doi.org/10.1068/p190705.

Van Selst, M., \& Jolicoeur, P. (1994). A solution to the effect of sample size on outlier elimination. The Q. J. Exp. Psychol. A Hum. Exp. Psychol., 47, 631-650. https://doi.org/10.1080/14640749408 401131.

Voss, A., Nagler, M., \& Lerche, V. (2013). Diffusion models in experimental psychology: A practical introduction. Exp. Psychol., 60, 385-402. https://doi.org/10.1027/1618-3169/a000218.

Voss, A., Voss, J., \& Lerche, V. (2015). Assessing cognitive processes with diffusion model analyses : a tutorial based on fast-dm-30. Front. Psychol., 6, 336. https://doi.org/10.3389/fpsyg.2015.00336.

Wearden, J. H., Edwards, H., Fakhri, M., \& Percival, A. (1998). Why "sounds are judged longer than lights": application of a model of the internal clock in humans. Q.J. Exp. Psychol. B, 51, 97-120. doi: $10.1080 / 713932672$. 
Wearden, J. H., Philpott, K., \& Win, T. (1999). Speeding up and (... relatively ...) slowing down an internal clock in humans. Behav. Proc., 46, 63-73. https://doi.org/10.1016/S0376-6357(99)00004-2. Wearden, J.H., Smith-Spark, J. H, Cousins, R., Edelstyn, N. M. J., Cody, F.W.J., \& O’ Boyle, D.J., (2014) Effect of click trains on duration estimates by people with Parkinson's disease. Q. J. Exp. Psychol., 62, 33-40. https://doi.org/10.1080/17470210802229047.

Wearden, J. H., Williams, E. A., \& Jones, L. A. (2017). What speeds up the internal clock? Effects of clicks and flicker on duration judgements and reaction time. Q. J. Exp. Psychol., 70, 488-503. https://doi.org/10.1080/17470218.2015.1135971.

White, C. N., Curl, R. A., \& Sloane, J. F. (2016). Using decision models to enhance investigations of individual differences in cognitive neuroscience. Front. Psychol., 7, 81. https://doi.org/10.3389/ fpsyg.2016.00081.

Wittmann, M. (2011). Moments in time. Front. Integr. Neurosci., 5, 66. https://doi.org/10.3389/ fnint.2011.00066. 